\title{
Vielfältige Sammlungsobjekte und ihre Archivalien in neuem Licht.
}

\section{Ein interdisziplinäres Forschungsprojekt zur Kunstkammer der Herzöge von Württemberg}

\author{
Von Fritz Fischer, Ingrid-Sibylle Hoffmann und MaAike van Rijn
}

Die Kunstkammer der Herzöge von Württemberg gehört mit fast 4.000 erhaltenen Objekten zu den bedeutendsten historischen Kunstkammern Europas und zeichnet sich durch eine besonders dichte archivalische und materielle Überlieferung aus. Erstmals wird die Kunstkammer in der Regierungszeit Herzog Friedrichs I. (reg. 1593-1608) erwähnt ${ }^{1}$. In ihren Anfängen spiegelt die Kunstkammer eine universalistische Sammlungsidee, die um 1600 zahlreiche Fürsten aufgriffen und in umfangreichen Kollektionen an ihren Höfen verwirklichten. Mehrfache Kriegsbedrohungen, sich wandelnde Sammlungsinteressen der Herzöge und geistesgeschichtliche Strömungen ebenso wie sich verändernde staatliche Strukturen nahmen in der Folge Einfluss auf die Sammlung. Dennoch bestand die herzogliche Kunstkammer über einen Zeitraum von mehr als 200 Jahren, von ihren Anfängen vor 1600 bis zur Übertragung in die staatliche württembergische Verwaltung im Jahr 1817, und lebt in ihren Nachfolgeinstitutionen bis heute fort.

So zählen die Objekte der ehemaligen herzoglichen Kunstkammer zu den wichtigsten Kernbeständen des Landesmuseums Württemberg. Die Kunstkammer ist gleichsam das Herzstück des Hauses und wird in der 2016 neu eröffneten Schausammlung „Wahre Schätze“ im 1. Obergeschoss des Alten Schlosses in Stuttgart umfänglich präsentiert ${ }^{2}$ (Abb.1). Einstige Kunstkammer-Bestände sind darüber hinaus auf verschiedene weitere Institutionen verteilt: völkerkundliche Objekte gelangten ins Linden-Museum, und Mineralien, Fossilien und Herbarien werden im Staatlichen Museum für Naturkunde in Stuttgart bewahrt. Weitere Pretiosen

1 Der erste Hinweis auf die Stuttgarter Kunstkammer befindet sich im Reisebericht des weit gereisten Basler Mediziners Felix Platter (1536-1614) aus dem Jahr 1596 und ist nachzulesen in: Valentin LöTscher (Hg.), Felix Platter: Tagebuch (Lebensbeschreibung) 1536-1567 (Basler Chroniken 10), Basel/Stuttgart 1976, S. 481.

${ }^{2}$ Dazu die Begleitbücher: Wahre Schätze. Antike - Kelten - Kunstkammer, hg. vom Landesmuseum Württemberg, 3 Bde., Ostfildern 2016. 
befinden sich in der Staatsgalerie Stuttgart, der Württembergischen Landesbibliothek, dem Hauptstaatsarchiv Stuttgart, in den Schlössern Ludwigsburg und Bebenhausen, im Privatbesitz des Hauses Württemberg sowie im Kunsthistorischen Museum Wien.

Trotz der 1976 veröffentlichten, grundlegenden Publikation von Werner Fleischhauer ${ }^{3}$ fehlte bislang eine umfassende wissenschaftliche Darstellung der württembergischen Kunstkammer und ihrer kulturgeschichtlichen Bedeutung. Eine umfangreiche Förderung durch die Deutsche Forschungsgemeinschaft (DFG) in den Jahren 2012 bis 2015 ermöglichte eine systematische Erschließung der Bestände und die Rekonstruktion der Sammlung. Durch die Zuordnung der erhaltenen Objekte zu den vornehmlich im Hauptstaatarchiv Stuttgart befindlichen historischen Inventaren, konnten unter anderem der jeweilige Eingang in die Kunstkammer sowie die sich teils im Laufe der Zeit wandelnde Verortung innerhalb der Sammlung rekonstruiert werden. Mittels intensiver Quellenstudien wurden ferner auch die Verluste greifbar, vor allem aber konnten die Rahmenbedingungen für die Entstehung und Entwicklung der württembergischen Kunstkammer präzisiert werden. Im Fokus standen die Bedeutung und repräsentative Funktion der Sammlung für die jeweiligen Herrscher. Gefragt wurde nach spezifischen Merkmalen, den verschiedenen Sammlungsstrategien sowie nach der kommunikativen Funktion der Bestände im Hinblick auf den Austausch von Geschenken und den kulturellen Transfer zwischen europäischen Fürstenhöfen. Von Interesse war schließlich, wer die Sammlung gesehen hat und wie sie rezipiert wurde.

Die im Jahr 2017 mit Erscheinen der dreibändigen Publikation ${ }^{4}$ sowie der Veröffentlichung der überlieferten Objekte im digitalen Kunstkammer-Katalog ${ }^{5}$ der Öffentlichkeit zugänglich gemachten Ergebnisse sollen im Folgenden skizziert werden.

\section{Grundlagen des Forschungsprojektes und zentrale Ergebnisse}

Bei der Rekonstruktion des Gesamtbestandes der Stuttgarter Kunstkammer war zu berücksichtigen, dass sich die zu bearbeitenden Objekte nicht nur im Landesmuseum Württemberg befinden, sondern Teile der Sammlung in weiteren Stuttgarter Institutionen sowie vereinzelt an anderen Orten bewahrt werden ${ }^{6}$. Außerdem

\footnotetext{
3 Werner Fleischhauer, Die Geschichte der Kunstkammer der Herzöge von Württemberg in Stuttgart, Stuttgart 1976.

${ }^{4}$ Die Kunstkammer der Herzöge von Württemberg. Bestand, Geschichte, Kontext, hg. vom Landesmuseum Württemberg, 3 Bde., Ulm 2017.

5 http://www.kunstkammer-stuttgart.de bzw. http://www.landesmuseum-stuttgart.de/ sammlungen/digitaler-katalog/kunstkammer/ (letzter Zugriff: 20.12.2017).

${ }^{6}$ Außer im Landesmuseum Württemberg befinden sich ehemals in der Kunstkammer präsentierte Objekte im Hauptstaatsarchiv Stuttgart, in der Württembergischen Landes-
} 
war damit zu rechnen, dass die Bestände nicht nur zum Fachbereich der Kunstgeschichte gehören, sondern auch zur Archäologie und Volkskunde sowie zu verschiedenen Disziplinen der Naturkunde und der Ethnologie. Das komplexe Forschungsvorhaben musste interdisziplinär von Wissenschaftlern der genannten Fächer und zusammen mit Archivaren, Bibliothekaren, Fotografen, Historikern, Museologen und Restauratoren angegangen werden. Unter Federführung des Landesmuseums Württemberg waren letztlich insgesamt weit über 50 Personen daran beteiligt. Gemeinsames Ziel war es, mit dem Abschluss des Projekts dem vor rund 40 Jahren von Werner Fleischhauer geäußerten Wunsch nach einer „Gesamtpublikation der Kunstkammer" zu entsprechen”.

Mit mehr als 120 überlieferten Inventaren und Inventarfragmenten gehört die Stuttgarter zu den am besten dokumentierten frühneuzeitlichen Kunstkammern. Den Kernbestand der archivalischen Überlieferung bildet ein im Hauptstaatsarchiv Stuttgart bewahrtes Korpus von Inventaren aus dem 17. bis frühen 19. Jahrhundert $^{8}$. Anhand dieser Quellen lassen sich die Entwicklung des Sammlungsbestandes, seine Gliederungssystematiken und teils auch seine räumliche Aufstellung dokumentieren. Der Kernbestand wird ergänzt durch weitere Teilinventare, Erwerbs- und Abgangsverzeichnisse sowie Schriftzeugnisse zur Verwaltung der Kunstkammer. Unentbehrliche Grundlage für das Forschungsprojekt war ein differenziertes Findbuch zu den für die Kunstkammer einschlägigen Archivalien im Hauptstaatsarchiv Stuttgart, das Dank der Förderung der Stiftung Kulturgut Baden-Württemberg erstellt werden konnte und online einsehbar ist ${ }^{9}$.

Stand Werner Fleischhauers Untersuchung am Beginn der neueren Kunstkammerforschung in der zweiten Hälfte des 20. Jahrhunderts, so konnten für das aktuelle Forschungsprojekt, insbesondere für die Frage nach dem Spezifischen der Stuttgarter Kunstkammer wie auch für die Bewertung einzelner Bestandsgruppen im zeitgenössischen Vergleich, eine ganze Reihe von in jüngerer Zeit erschienenen Untersuchungen $\mathrm{zu}$ anderen fürstlichen Kunstkammern herangezogen werden. Dabei waren besonders die Arbeiten zu den Münchner und Dresdner Sammlungen sowie die Untersuchungen zur Braunschweiger, Gottorfer sowie zur Gothaer Kunstkammer aufschlussreich ${ }^{10}$.

bibliothek, im Staatlichen Museum für Naturkunde Stuttgart, im Linden-Museum Stuttgart - Staatliches Museum für Völkerkunde, in der Staatsgalerie Stuttgart, bei den Staatlichen Schlössern und Gärten Baden-Württemberg, im Kunsthistorischen Museum Wien, in der Schatzkammer der Münchner Residenz sowie in Privatbesitz.

7 Fleischinauer (wie Anm. 3), S.XI.

${ }^{8}$ HStA Stuttgart, Bestand A 20 a.

${ }^{9}$ Vgl. das Online-Findbuch von Niklas Konzen unter https://www2.landesarchivbw.de/ofs21/olf/einfueh.php?bestand=2988\#_4 (letzter Zugriff: 21.12.2017).

10 Wichtige Referenzwerke für die Einordnung der Stuttgarter Kunstkammer in den zeitgenössischen Kontext sind: Gottorf im Glanz des Barock. Kunst und Kultur am Schleswiger Hof 1544-1713, Katalog der Ausstellung des Schleswig-Holsteinischen Landesmuseums auf Schloss Gottorf, 4 Bde., Schleswig 1997; Weltenharmonie. Die Kunstkammer und 
Das Projekt startete unter der Annahme, dass sich etwa 1.700 Objekte aus der württembergischen Kunstkammer erhalten hätten. Durch die institutionenübergreifende Erforschung der Bestände und die umfangreiche Einbeziehung der Archivalien durch die jeweiligen Bearbeiterinnen und Bearbeiter der einzelnen Objektgruppen ist mittlerweile von etwa 4.000 überlieferten Stücken auszugehen. Vor allem auf dem Gebiet der Münzen und Medaillen waren erhebliche Zuwächse zu verzeichnen. Zu Beginn des Projekts waren nur wenige der ehemaligen Kunstkammerobjekte eingehender wissenschaftlich bearbeitet. Nun werden nicht nur eine Vielzahl einzelner Werke, sondern auch ganze Objektgruppen erstmals veröffentlicht: $\mathrm{Zu}$ nennen sind beispielsweise die Gemmen, die Münzen, die wissenschaftlichen Instrumente, die technischen Modelle, das Rubinglas, die Bronzen, die Möbel, die Musikinstrumente, die Gemälde und die Waffen. Obwohl im Rahmen des Projekts im Bereich Restaurierung hauptsächlich konservatorische Maßnahmen - ermöglicht durch die Wüstenrot Stiftung - durchgeführt wurden, und nicht die Möglichkeiten für aufwendigere Untersuchungen gegeben waren, konnten auch hier punktuell neue Erkenntnisse zu den verwendeten Materialen oder zu Herstellungsverfahren gewonnen werden.

Um sich ein annähernd stimmiges Bild von der Stuttgarter Kunstkammer zu machen, galt es ferner nicht nur die erhaltenen Objekte zu bearbeiten, sondern sich überdies anhand der historischen Inventare die Vielzahl von Gegenständen zu vergegenwärtigen, die nicht erhalten sind oder deren ehemalige Zugehörigkeit zum Bestand der Stuttgarter Kunstkammer sich bislang nicht nachweisen lässt. So konnten etwa anhand der Inventareinträge der weitgehend verlorene Bestand von einst etwa 4.000 grafischen Blättern rekonstruiert werden und detailliertere Vorstellungen von den einstigen Gemälde- und Waffensammlungen gewonnen werden. Außerdem vermitteln die Archivalien eine Idee davon, dass ganze Sammlungsbereiche komplett verloren sind, unter anderem Gegenstände aus extrem vergänglichen, organischen Materialien wie Textilien oder empfindliche Naturalia wie präparierte Tiere, Gipskopien bekannter Kunstwerke oder auch Kostbarkeiten, die eingeschmolzen werden konnten, wie Tafelgeschirr aus Gold und Silber.

In ihren Merkmalen und in der Entwicklung, die sie während ihres über 200-jährigen Bestehens nahm, unterscheidet sich die Stuttgarter nicht grundsätzlich von

die Ordnung des Wissens, Katalog der Ausstellung im Herzog Anton-Ulrich-Museum Braunschweig, Braunschweig 2000; Dominik Collet, Die Welt in der Stube. Begegnungen mit Außereuropa in Kunstkammern der Frühen Neuzeit, Göttingen 2007; Willibald SAUERLÄnder (Hg.), Die Münchner Kunstkammer, 3 Bde., München 2008; Jørgen HeIn, The Treasure Collection at Rosenborg Castle. The inventories of 1696 and 1718, royal heritage and collecting in Denmark-Norway 1500-1900, 3 Bde., Kopenhagen 2009; Dirk SYNDram/ Martina Minning (Hg.), Die kurfürstlich-sächsische Kunstkammer in Dresden. Geschichte einer Sammlung, Dresden 2012; Barbara Marx/Peter Plassmeyer (Hg.), Sehen und Staunen. Die Dresdner Kunstkammer von 1640. Dokumentation der Objekte in Dresdner Sammlungen, Berlin u. a. 2014. 
den anderen großen Kunstkammern. Sie verdankte ihr Profil in geringerem Maße den Vorlieben einzelner Sammlerpersönlichkeiten, prägender waren Hinzugewinne durch Erbschaften, die jeweiligen finanziellen Möglichkeiten, die aktuellen Angebote auf dem Kunstmarkt und natürlich auch die kriegsbedingten Verluste. Auch die Herzöge von Württemberg wollten in ihrer Kunstkammer die Geschichte ihrer Dynastie und ihres Landes dargestellt wissen. Und wie an anderen Orten war die Kunstkammer in Stuttgart ein Teil der sich stets wandelnden höfischen Repräsentation, die den Ruhm des Fürstenhauses, der Residenz und des Landes mehren musste und daher stetig modernisiert wurde.

Wie bei anderen Kunstkammern spiegeln die Inventare auch in Stuttgart die Entwicklung und die Dokumentation der Sammlung wider. Dabei ist jedoch der Informationswert zu den einzelnen Stücken eng begrenzt. Über ihre Herkunft, ihre damalige kunsthistorische Einordnung oder ihre ursprüngliche Funktion erfährt man in den Inventaren in der Regel wenig. Dennoch sind die Inventare die Grundlage, auf der das bisher entworfene Bild von der Kunstkammer der Herzöge von Württemberg ergänzt werden kann: Einige Sammlungsbereiche wie etwa die archäologischen Bodenfunde oder die Naturalia spielten quantitativ und qualitativ eine größere Rolle als bisher angenommen, manche wie beispielsweise die Bücher oder die Druckgraphik fahnden erstmals Beachtung.

\section{Geschichte und Kontext der herzoglichen Kunstkammer ${ }^{11}$}

In der Anfangsphase, also vom Ende des 16. Jahrhunderts bis zur Schlacht von Nördlingen (1634), spiegelte die württembergische Kunstkammer den Ehrgeiz und den weiten Horizont ihres Gründers Herzog Friedrich I. (reg.1593-1608) wider (Abb. 2). Ähnlich wie andere fürstliche Initiatoren von Kunstkammern im 16. Jahrhundert hatte er den Anspruch, universell - Macrocosmos in Microcosmo - zu sammeln. Besonders suchte er nach Gegenständen, mit denen die Besucher zum Staunen über die Besonderheiten der Welt und die handwerklichen Möglichkeiten gebracht wurden: Exotika, Naturalia und Automaten. Sein Sohn und Nachfolger, der prächtig Hof haltende Herzog Johann Friedrich (reg. 1608-1628) prunkte mit Kostbarkeiten aus den edelsten Materialien. Er kaufte bestes zeitgenössisches Kunsthandwerk oder ließ es von den führenden Handwerkern an seinem Hof herstellen. Geschmackliches Vorbild war dabei die italienische Kunst. Gezeigt wurden die Schätze in drei Räumen unter dem Dach des Schlosses, zu

11 Detaillierte Einblicke in Geschichte und Kontext der Kunstkammer der Herzöge von Württemberg geben in der 2017 erschienenen Publikation zur württembergischen Kunstkammer (wie Anm. 4) die Beiträge von Carola FEY „Die Geschichte der württembergischen Kunstkammer“ (Bd.1, S.72-101) und „Ordnung, Präsentation und Kommunikation“ (Bd.1, S.102-131). Hier siehe auch die entsprechenden Nachweise für die folgenden Ausführungen. 
losen Gruppen zusammengestellt auf Tischen, Bänken und Schaubüffets - so wie das auch andernorts, etwa in Gotha, Dresden oder München, üblich war. Zu sehen bekam die Kunstkammer die höfische Elite des Reiches.

Nach der Katastrophe des Dreißigjährigen Krieges, die zum weitgehenden Verlust der frühen Kunstkammerbestände geführt hatte, kam es zu einem Neuanfang mit Innovationen. Die treibende Kraft war der Herzog selbst: Kein Zweiter hat das Profil der württembergischen Kunstkammer so geprägt wie Eberhard III. (reg. 1628 -1674). Er holte die Sammlung aus dem abgelegenen Ort unter dem Dach des Schlosses hervor. Fortan wurde sie im Alten Lusthaus gezeigt, einem eigenen Haus mitten im Lustgarten, der vor allem wegen des legendären Neuen Lusthauses und der spektakulären Grotte eine touristische Attraktion Stuttgarts und des ganzen Reiches war. Der Herzog erreichte damit eine bessere Zugänglichkeit der Kunstkammer, die offenbar auch dazu dienen sollte, im Rahmen seiner demonstrativ prachtvollen Hofhaltung ein Zeichen für die nach den Kriegszerstörungen wieder erstarkte Stellung Württembergs im Reich zu setzen.

Aus den schriftlichen Quellen ist herauszulesen, wie sehr sich Eberhard III. für das Aufblühen seiner Kunstkammer einsetzte: Er gab Kostbarkeiten aus seinem eigenen Besitz in die Sammlung, er bestimmte testamentarisch die Bestände der Kunstkammer als unveräußerlich, er schrieb den Superintendenten und Pfarrern, sie mögen im Land Gegenstände ausfindig machen, die man in der Stuttgarter Kunstkammer zeigen könnte. Er kaufte auf dem Kunstmarkt, vor allem in Augsburg und Nürnberg, Kupferstiche, Kristallgeschirre, Gemälde, Skulpturen, optische Geräte und er bemühte sich um den Ankauf ganzer Sammlungen. Sein besonderes Interesse galt neben der Goldschmiedekunst dem landeshistorisch Relevanten, also den Münzen und den Bodenfunden aus der Region.

Eberhard III. wollte auch die Verwaltung der Kunstkammer grundlegend reformieren. Dem zuständigen Kunstkämmerer legte er nahe, sich mit Gelehrten und anderen Sammlern auszutauschen. Die Inventare sollten nicht nur der Wiederauffindung der Gegenstände dienen, die Objekte sollten darin vielmehr historiografisch gedeutet sowie in Text und Bild dokumentiert werden.

Auch hinsichtlich der Präsentation der Sammlung begann unter Eberhard III. die Musealisierung der Kunstkammer: Die Gegenstände wurden nach Kriterien wie Material oder Funktion in Gruppen geordnet und größtenteils in Schränken aufbewahrt. Zu dieser Entwicklung hin zu mehr Ordnung gehörte auch, dass der Herzog ganze Sammlungsteile aussonderte, wie dies auch schon andernorts, etwa in Wien, durchgeführt worden war, und somit den ersten Schritt zur Auflösung der Kunstkammer in die Wege leitete. So ließ er die durch den Auszug der Kunstkammer frei gewordenen Räume im Schloss renovieren und dort einen Raum einrichten, in dem nur Gemälde und Modelle ausgestellt wurden. Die neue, in der Kunstkammer wie in der gesamten Hofhaltung zur Schau gestellte Ordnung sollte letztlich die gut geregelte Regierung des Herrschers im Zeitalter des aufkommenden Absolutismus veranschaulichen. 


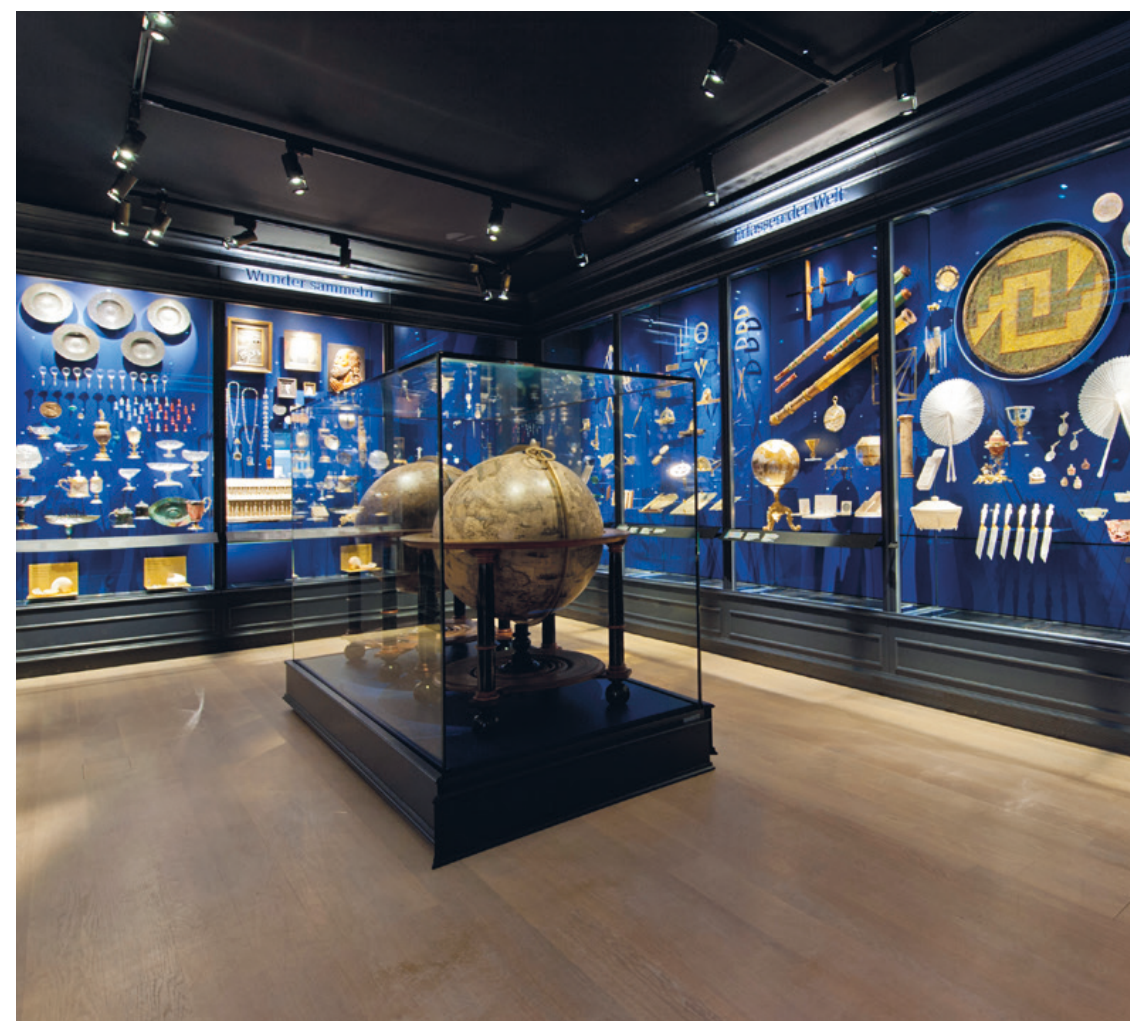

Abb. 1: Blick in das sogenannte Studiolo in der Schausammlung „Wahre Schätze“ im Landesmuseum Württemberg (Altes Schloss, Stuttgart). 


\section{FRIDERICVS DVX WIRTENBERG.}

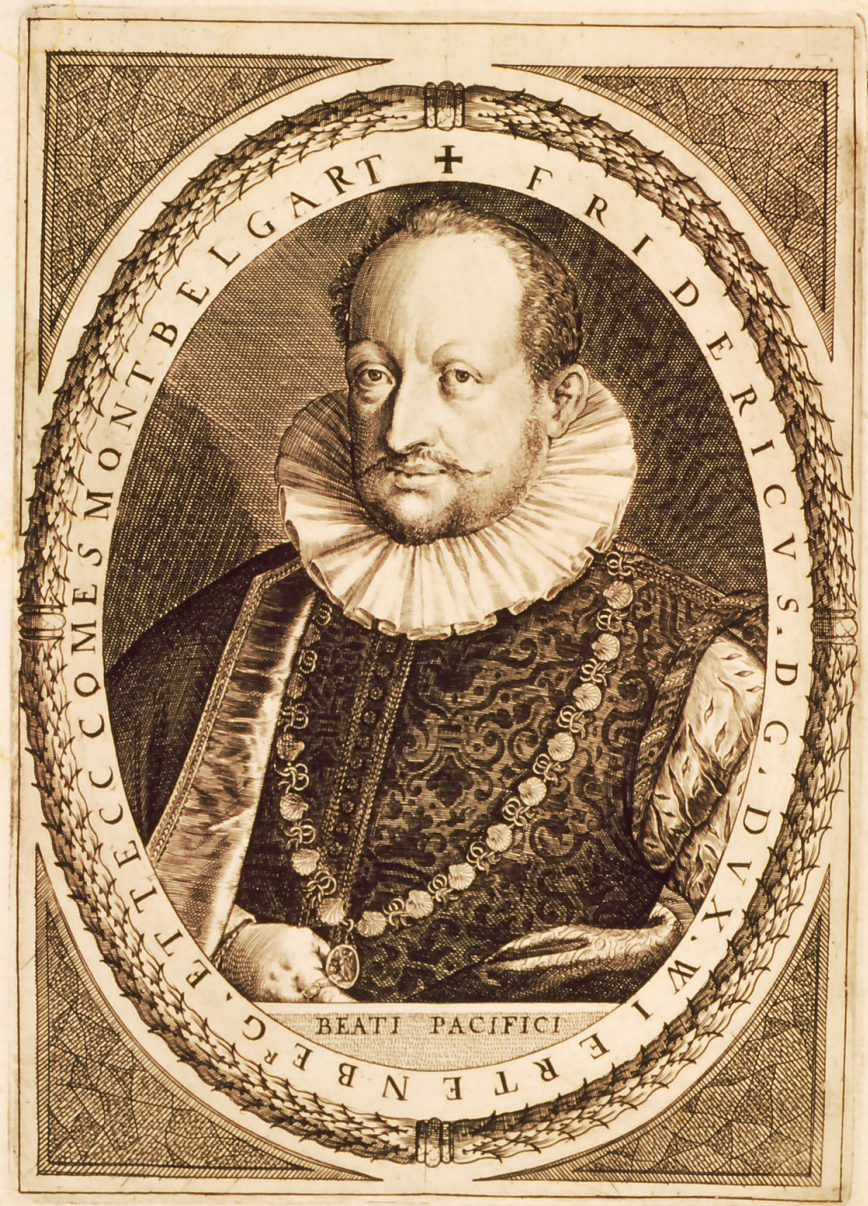

Virtenbergenfi qui Dux dat iura colono, Hec facies, hac eft buius imago Ducis;

Sanguine preluftri qui Alemanna aborigine cretus, Viniferos patria nunc moderatur agros:

Pacis, of armorum, tuxta atis aptus ad artes, Pacis, vtiprefert nomine, diues opumeft.

Abb. 2: Porträt Herzog Friedrichs I. von Württemberg (reg. 1593-1608). 


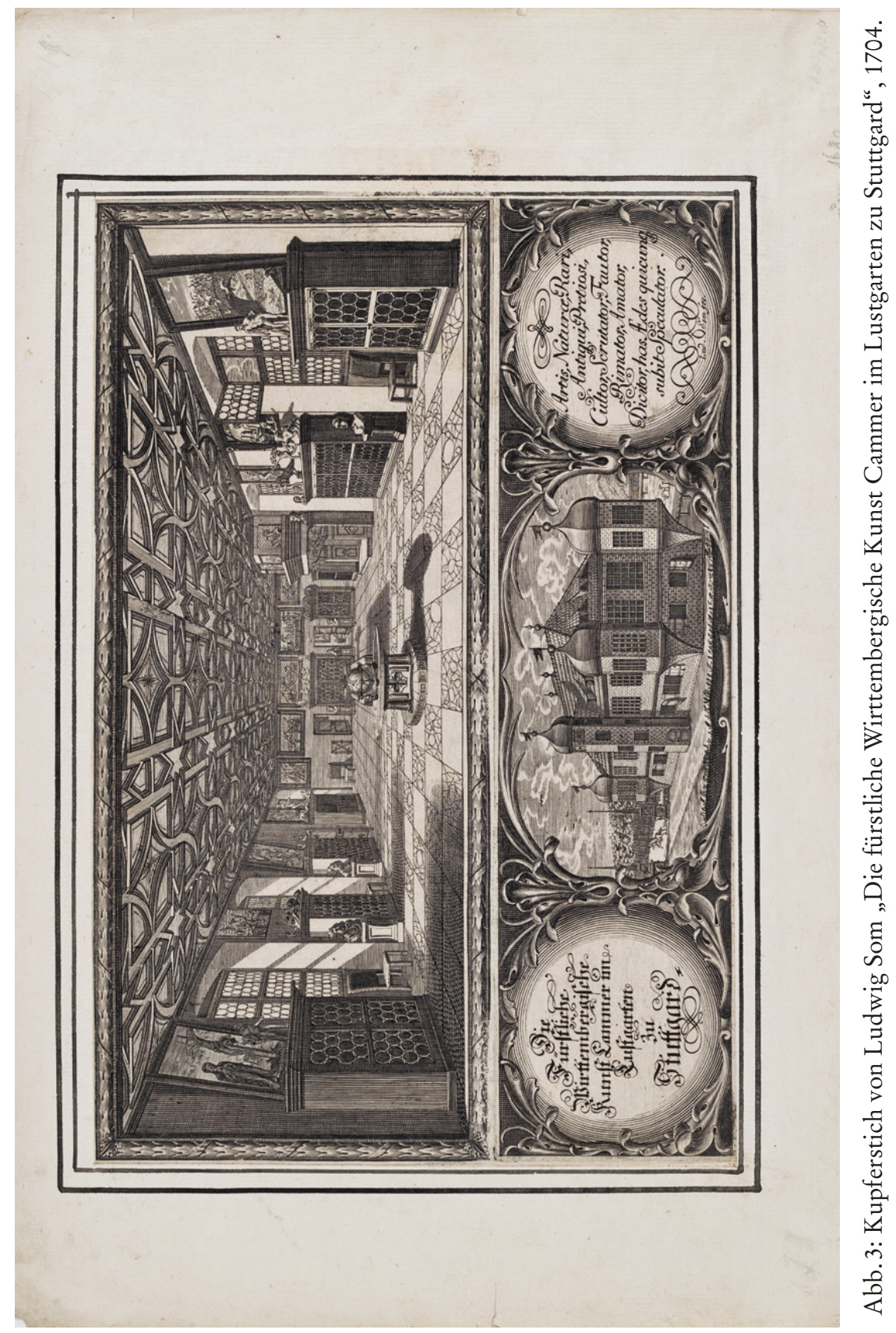




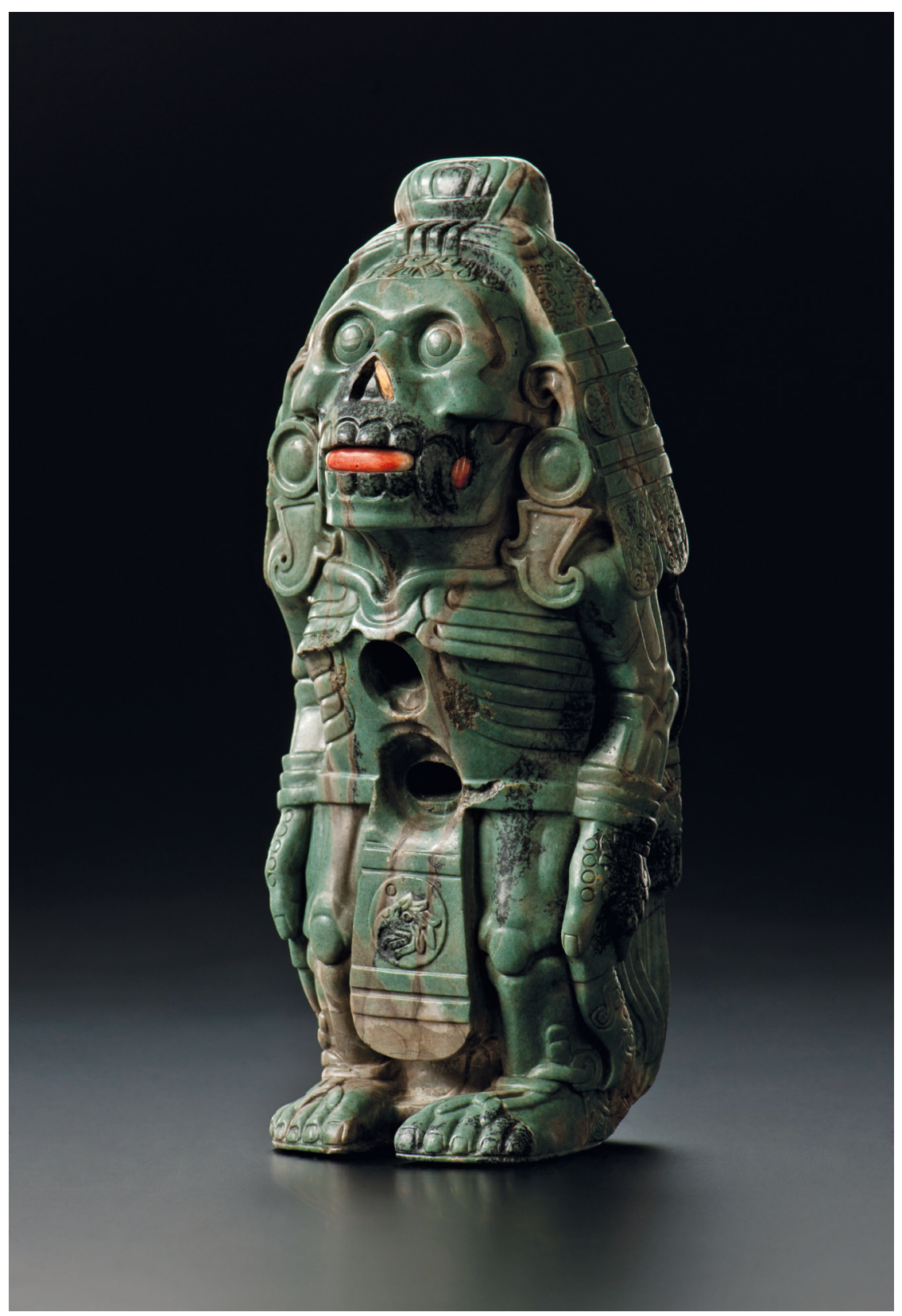

Abb. 4: Götterfigur der Azteken. Mexiko, 1500-1520. 


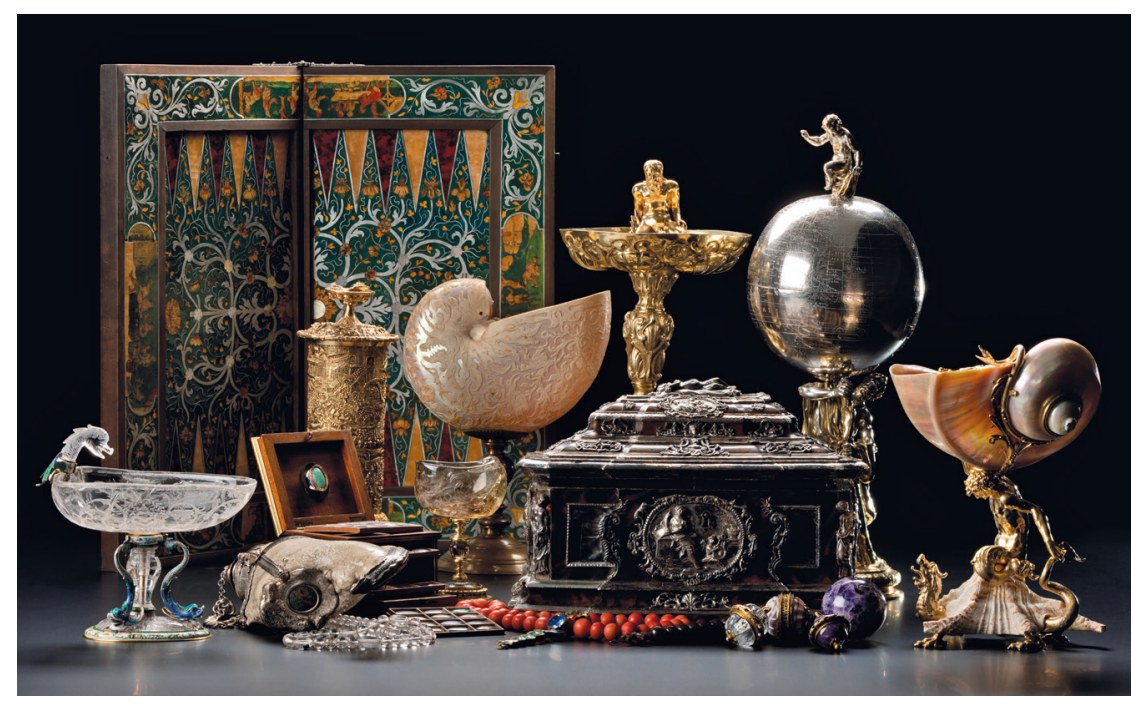

Abb. 5: Ensemble verschiedener Pretiosen aus der württembergischen Kunstkammer.

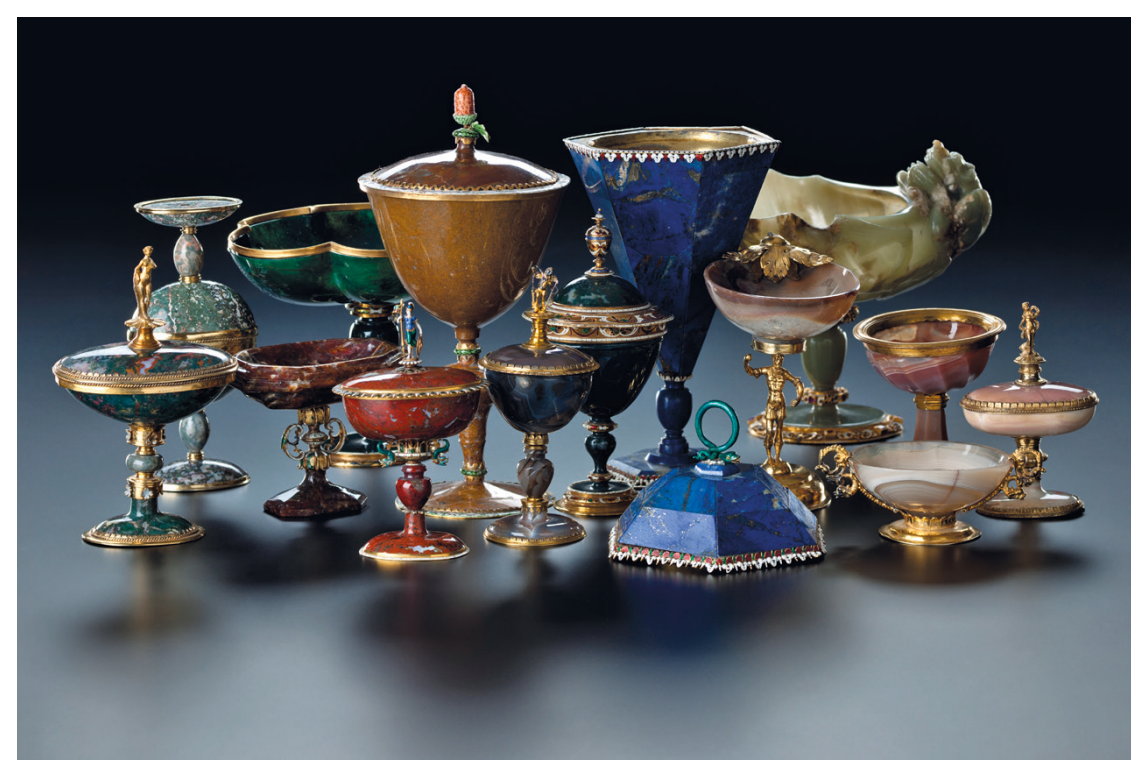

Abb. 6: Ensemble von Steinschnittarbeiten aus der württembergischen Kunstkammer, 17./18. Jahrhundert. 
44.

\section{Las untentte. Siefacs.}

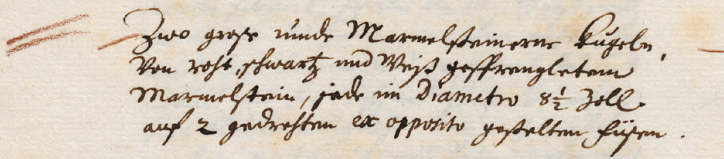

Transfercint, fatt min Diamotns min fouls wigh

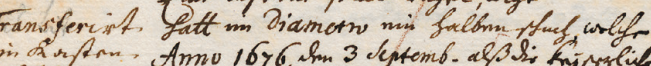

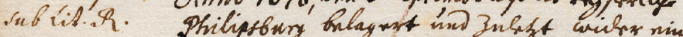

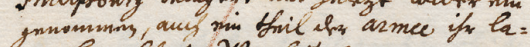

gro bryon Clost. Wagfoingel ynnomenum,

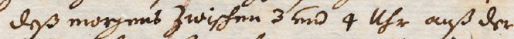

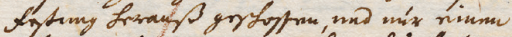

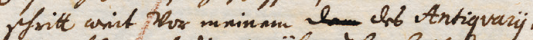

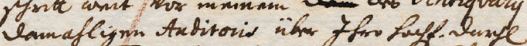

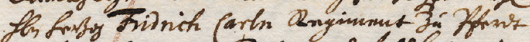

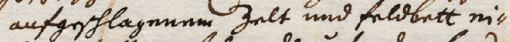

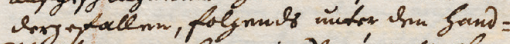

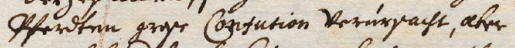

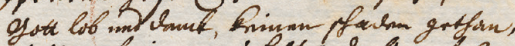

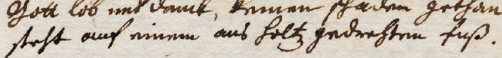

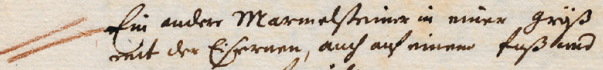
cx oppotito anfyraigt.

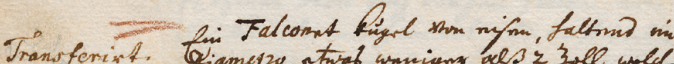
in toratim. subtil.

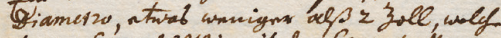

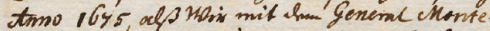

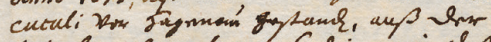

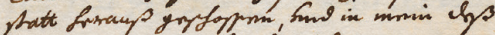
Antrigrariy jnet ofm hung chppres pange

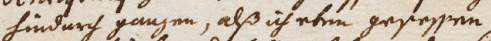

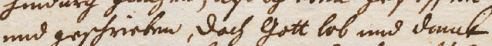

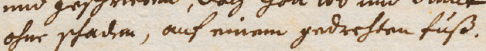

Abb. 7: Eine Seite aus den Inventaren Johann Schuckards, Unterfaszikel 2:

Kasten E und F, um 1705/23 (HStA Stuttgart, A 20 a Bü 17, S. 44). 
Herzog Eberhard Ludwig (reg. 1693-1733) ging, wie auch seine Nachfolger, weniger engagiert, sondern eher pragmatisch mit der Kunstkammer um. Sie war Teil seiner höfischen Repräsentation, bei der auch der Verweis auf die historischen Leistungen der eigenen Dynastie eine große Rolle spielte. Aus diesem Grund rettete er die Sammlungen seiner Vorfahren und Verwandten vor dem Untergang. Er kaufte u. a. die Sammlungen der Nebenlinie Württemberg-Neuenstadt, die Antiquitäten des Mömpelgarder Zweiges und übernahm Pretiosen aus dem Nachlass der Herzogin Sibylla von Württemberg-Mömpelgard (1620-1707). Die Kunstkammer begann nun ein Aufbewahrungsort für Gegenstände zu werden, die wie eine Reihe von älteren astronomischen Instrumenten oder säkularisiertes Kirchengut ihre ursprüngliche Funktion verloren hatten und nun zu historischen Studienobjekten wurden.

Welchen Charakter die Sammlung zu Beginn des 18. Jahrhunderts hatte oder haben sollte, belegt ein Stich mit einem Blick in die Kunstkammer, den Eberhard Ludwig 1704 von Ludwig Som (tätig um 1704-1725) anfertigen ließ (Abb.3). Er stellt die einzige Bildquelle zur württembergischen Kunstkammer dar und bietet die Möglichkeit, die in ihm enthaltenen Informationen mit denen abzugleichen, die uns die schriftlichen Quellen überliefern. Der Stich präsentiert den Ausstellungsraum im besten Licht: besucherfreundlich eingerichtet, klar gegliedert, hell, gut belüftet und zweckmäßig möbliert. Das Gros der kleineren Gegenstände wird in verglasten Schränken aufbewahrt, in die man hineinsehen kann. Will der Besucher die Gegenstände herausholen und aus der Nähe betrachten, so stehen dazu Tische und Stühle bereit. Die größeren Gegenstände, einige Skulpturen und Naturalia, stehen oben auf den Schränken, auf den freien Wandstücken darüber hängen die großformatigen Gemälde.

Die schriftlichen Quellen vermitteln ein ganz anderes Bild vom damaligen Aussehen der Kunstkammer. Laut dem von Johann Schuckard (1640-1725, tätig: 1690-1725) relativ zeitgleich mit dem Stich erstellten Inventar ${ }^{12}$ (Abb.7), hingen auch in jener Zeit noch Tierpräparate von der Decke und die Wände waren nicht im Stil der aufkommenden Gemäldegalerien dekoriert, sondern mit einer Fülle von weiteren Gegenständen, etwa mit Kupferstichen und exotischen Waffen, bedeckt.

Der Stich trägt eine in Latein verfasste Beischrift, die deutlich macht, wer in der Kunstkammer des Herzogs erwartet wird: der Verehrer, Erforscher, Gönner, Ergründer, Liebhaber und Kundige von Kunst, Natur, Seltenheiten, Antiken und Kostbarkeiten. Das Spektrum der diesen unterschiedlichen Besuchern dargebotenen Gegenstände bleibt noch universell, doch werden sie in stärker systematisierter Form als zuvor präsentiert. Ähnliche Tendenzen wie in der idealisierten Stuttgarter Ansicht lassen sich in Dresden, Gottorf und Gotha beobachten.

Die angestrebte neuartige Ordnung der Kunstkammer spiegelt sich auch in der Systematik der zeitgenössischen Inventare. Schuckard hatte geplant, ein neues Ge-

12 HStA Stuttgart A 20 a Bü 17. 
samtinventar der Kunstkammerbestände anzufertigen. Es sollte aus drei Teilen bestehen. Im ersten sollten die Gegenstände nach Objektarten und Materialien geordnet, im zweiten nach Lagerorten sortiert sein. Der dritte Teil sollte ein alphabetisches Verzeichnis beinhalten. Realisiert hat Schuckard nur den zweiten Teil, das nach Orten gelistete Inventar. Und nur bei der Erfassung der römischen Steindenkmäler erreicht er jene schon von Eberhard III. eingeforderte Wissenschaftlichkeit, etwa durch den Verweis auf spätantike Literatur.

Im weiteren Verlauf des 18. Jahrhunderts setzte sich die Aufgliederung der Bestände fort, unter Herzog Carl Eugen (reg. 1737-1793) beschleunigte sich die Auflösung der württembergischen Kunstkammer: Die Kupferstiche und Zeichnungen wurden ins Ludwigsburger Schloss gebracht, ebenso ein Teil der Bronzen. Mit Beständen der Kunstkammer entstanden in Ludwigsburg eine Gemäldegalerie und ein mathematisches Kabinett. Pretiosen aus der Kunstkammer schmückten die Gemächer der herzoglichen Mätresse und späteren Gemahlin des Herzogs. Bücher aus der Kunstkammer bildeten den Grundstock für die neu gegründete Herzogliche Bibliothek. Große Sammlungsteile wurden als Anschauungsmaterial in die Hohe Carlsschule verbracht.

1791 wurde das Naturalienkabinett, dem die Professoren der naturkundlichen Fächer der Hohen Carlsschule vorstanden, von der Kunstkammer abgetrennt. Für Letztere sollte weiterhin der bisherige Antiquar Johann Friedrich Vischer (1726-1811, tätig 1768/69-1791) zuständig sein. In einem Schreiben an den Herzog fragte er jedoch, ob der verbleibende Rest der Kunstkammer, der schon ohnehin nur noch in Notunterkünften gezeigt wurde, überhaupt seine weitere Anstellung rechtfertige.

Gleichzeitig blieb die Frage nach der Öffnung der Sammlungen für ein möglichst breites Publikum virulent. Jedenfalls forderte der Diplomat und Mineraloge Heinrich von Struve (1772-1851), als er 1807 Teile der aus der Hohen Carlsschule ins Alte Schloss umgezogenen Naturalia bewundern durfte, dass diese Sammlung öffentlich zugänglich gemacht werden und an bestimmten Tagen kostenlos zu besichtigen sein sollte.

1817 löste König Wilhelm I. (reg. 1816-1864) die Kunstkammer aus der herzoglichen Verwaltung und übergab sie der Obhut des Staates. Nicht länger auf die herrschende Dynastie bezogen, begann damit eine neue Geschichte, die des späteren Landesmuseums Württemberg.

\section{Die Veröffentlichung der Forschungsergebnisse}

Um die Forschungen der Öffentlichkeit zugänglich zu machen, wurden zwei Medien gewählt: die bereits erwähnte gedruckte dreibändige Publikation sowie der Online-Katalog. Der gedruckte Katalog gliedert sich in drei Teile: Der erste enthält übergreifende kulturhistorische Aufsätze zu Geschichte und Kontext der würt- 
tembergischen Kunstkammer, der zweite ist der Darstellung des überlieferten Bestandes - gegliedert nach Sammlungsbereichen - gewidmet. Jeder Komplex wird dabei zunächst durch einen Essay eingeleitet. Darauf folgen konzentrierte Beschreibungen von ausgewählten Objekten, wobei von den insgesamt fast 4.000 überlieferten Objekten rund 400 in 337 Katalognummern behandelt werden. Diese liefern Basisinformationen, meist einen kurzen Zustandsbericht, referieren die Forschungslage und stellen das Objekt in seinen Kontext innerhalb der Kunstkammer. Am Ende werden die Quellen zitiert, mit denen sich nachweisen lässt, dass der Gegenstand ehemals im Bestand der Kunstkammer war. Ein umfangreiches Orts- und Personenregister, ein Quellenverzeichnis, das erstmals alle überlieferten Inventare der württembergischen Kunstkammer aus dem 17. und 18. Jahrhundert auflistet und ein umfassendes Literaturverzeichnis runden - im dritten Band zusammengefasst - die Veröffentlichung ab.

Der Online-Katalog enthält Basis-Informationen zu allen erhaltenen Objekten der Kunstkammer, inklusive Abbildung, einen kurzen erläuternden Text sowie die Inventareinträge, die den Gegenstand als Teil der Kunstkammer ausweisen. Zudem ist über die Homepage des Landesmuseums Württemberg eine vollständige Transkription des Inventars von Johann Schuckard (1640-1725, tätig 1690-1725) einzusehen. ${ }^{13}$ Das Inventar entstand, als die Kunstkammer ihren Höhepunkt erreicht hatte. Es war strukturell vorbildlich für alle später angelegten Inventare. Nach den Aufbewahrungsorten der Gegenstände gegliedert und diese relativ ausführlich beschreibend, gibt das Inventar aufschlussreiche Einblicke in die Bestände der württembergischen Kunstkammer zur Zeit Herzog Eberhard Ludwigs (reg. 1693-1733).

$\mathrm{Zu}$ wünschen bleibt, dass die Kunstkammer der württembergischen Herzöge mit der Veröffentlichung der Erkenntnisse des mehrjährigen interdisziplinären Projektes in Print- und Onlinekatalog innerhalb der Forschung zu frühneuzeitlichen Kunstkammern einen neuen Stellenwert erhält. Ferner mögen von der spannenden Geschichte der Kunstkammer, ihrem wechselvollen Schicksal und den sich wandelnden Kontexten der Sammlung, aber auch den höchst faszinierenden Objektgeschichten weitere Untersuchungen zu den vielfältigen Aspekten der Stuttgarter Sammlungen sowie zum kulturellen Leben in der frühneuzeitlichen Residenzstadt angestoßen werden.

${ }^{13}$ http://www.landesmuseum-stuttgart.de/sammlungen/forschung/kunstkammer/transkription-kunstkammerinventare/ (letzter Zugriff: 21.12.2017). 
Abbildungsvorlagen:

Abb. 3: Ludwigburg Museum

Abb. 7: Landesarchiv Baden-Württemberg, Hauptstaatsarchiv Stuttgart

Alle weiteren Abbildungen: Landesmuseum Württemberg, Stuttgart (Foto: Hendrik Zwietasch) 